\title{
La Matemática Paleobabilónica
}

\author{
Manuel Pérez Rodriguez *
}

\begin{abstract}
RESUMEN ABSTRACT
La Matemática Paleobabilónica crece en la actividad de las escuelas de escribas. Resuelve problemas con valores numéricos concretos; es una matemática de cálculo. Su papel histórico puede recordar nuestros propios procesos de aprendizaje, en la primera etapa, cuando a través del uso de fórmulas con valores numéricos concretos, aprendimos a expresarlas de modo abstracto. Más aún, los temas de los que se ocupó recuerdan los temas de nuestros primeros aprendizajes en matemáticas. La actividad con el Teorema de Tales, gran parte, al menos, del Teorema de Pitágoras, los métodos y fórmulas básicas del Algebra..., un sistema de cálculo, posicional, que sólo fue superado en el Renacimiento, fueron creados en aquella escuela.

Old Babylonian Mathematics developed in the activity of escribal education. Solving problems with numeric values, is a computing mathematic. Historically, can remember us our own learning processes, in the first stage, when through the use of formulas with numeric values, we learned to express in an abstract way. Also, the problems that interested babylonian mathematics remember those that we worked out in our first educational activities in mathematics. Computations with the Thale's theorem, great part, at least, of Pythagora's, the basic methods and formulas of Algebra..., the computation system, positional, that is only improved in the Renaissance, were created in that school.
\end{abstract}

* Licenciado en Ciencias Exactas. 


\section{INTRODUCCIÓN}

En las escuelas de escribas, credas ${ }^{1}$ en el transcurso de la dinástia Ur III bajo el reinado de Shulgi (2084-2047 a.C.), Matemáticas era una asignatura del currículum escolar. La formación del alumno en el oficio de escriba, oficio que tenía el estatus social, junto con los sacerdotes, de «Población dependiente de los templos e integrado en ellos política $y$ económicamente" "2, la actividad escolar, con la dedicación de los profesores, crearian un nivel cultural nuevo alentado por el mismo Rey Shulgi: «...En dos himnos sumerios de autoglorificación, el Rey Shulgi enfatizaba sus habilidades matemáticas en los siguientes pasajes:

"Al lugar donde el hombre va a estudiar el arte del escriba, (ó sea), en el lugar de aprendizaje de las artes secretas, la resta, la suma, contar y contabilizar, él completa"...» ${ }^{3}$.

En los siglos posteriores, transcurso del período Paleobabilónico (20041595 a.C), tiene lugar un gran desarrollo de procedimientos matemáticos. De éste período se conservan en diferentes Museos tablillas de barro con problemas de Matemáticas. Estas tablillas han sido clasificadas ${ }^{4}$ en dos grandes grupos: los textos-tabla y los textos-problema.

El grupo de los textos-tabla está formado por tablas de multiplicar, tablas de recíprocos (que se utilizaban para la división), tablas de cuadrados, tablas de raíces cuadradas, cubos, etc... y tablas metrológicas que realizan conversiones de unidades.

En el grupo de los textos-problema tenemos diversos problemas de Geometría y Algebra, unas veces en ejercicios con enunciados «matemáticos puros" y otras con enunciados de «matemáticas de aplicación»; con objetos como la construcción de canales, excavaciones, etc. La calidad de un buen número de ejercicios de los textos-problema, contrasta con la simplicidad concreta de los textos-tabla. Para O. Neugebauer, principal investigador de éstos textos,

«La diferencia en uso entre textos-tabla y texfos-problema radica sólo en el hecho que los textos-tabla ordinarios pertenecian a un nivel mucho más bajo de educación del escriba y tenían amplio uso fuera de las escuelas, mientras los textos-problema son esencialmente una manifestación de una educación superior e indudablemente eran escritos y comprendidos sólo por un grupo muy reducido de escribas». (MCT, pág. 1.)

\footnotetext{
KRAMER, S.N. "The Sumerians; their history, culture and character». Pág. 235.

VÁzQUEZ Hovs, A.M. «Historia Antigua Universal l». UNED. Madrid 1996. Pág. 84.

Nemet-Nejat CMTREM. Pág. 9.

Neugebauer y Sachs. MCT. Pág. 1.
} 
La actividad escolar reflejada en los textos capacitaba en tres grandes áreas:

a) El conocimiento de las técnicas del sistema de cálculo para realizar las operaciones aritméticas: suma, resta, multiplicación (tablas de multiplicar) y división (tablas de recíprocos), y la realización de conversiones de unidades en el sistema metrológico (tablas metrológicas).

b) El conocimiento de los conceptos y procedimientos en «matemáticas de aplicación», basadas en el uso de conversiones de unidades en el sistema metrológico (tablas metrológicas).

c) El conocimiento de procedimientos de Algebra y de Geometría.

En Geometría, cálculos de áreas y volúmenes en variados ejercicios. Aplicaciones de la semejanza de formas geométricas (Teorema de Tales). La aplicación del Teorema de Pitágoras (al menos a triángulos rectánculos semejantes al de lados $3,4,5) \ldots$

d) El conocimiento del lenguaje específico necesario, pues con la actividad matemática se crea un lenguaje Matemático, para describir operaciones (como «3 es la raíz cúbica de 27 \#...), para describir conceptos (asignación...), describir formas geométricas, etc.

\section{EL SISTEMA SEXAGESIMAL POSICIONAL}

«Con los Griegos, y después con los Arabes, el sistema sexagesimal ha sido un sistema académico de numeración, usado por los astrónomos. Todavía nos servimos de éste sistema para la medición de arcos o ángulos y la medición del tiempo" 5 .

El Sistema Sexagesimal Posicional utiliza dos signos para la representación del número:

I para la unidad y < para la decena. El número de escribe posicionalmente en base 60:

K<lll es el número sexagesimal 1,23

su valor decimal en el orden de la última cifra, 23 , es $1 \cdot 60+23=83$. En palabras actuales, 1 minuto y 23 segundos son 83 segundos.

5 Thureau-Dangin. SHSS. Pág. 95. 


\section{El origen del sistema}

Thureau-Dangin, en SHSS, cita a Neugebauer como autor de la propuesta de que el origen del sistema númerico sexagesimal es el sistema metrológico. El Sistema Metrológico Sumerio tenía relaciones variadas: sexagesimales, decimales y de docena, pero, ya en la época de los metales, cuando se crea la unidad de peso ma-na, la relación sexagesimal debía ser dominante ${ }^{6}$. Las unidades de longitud, como las demás unidades del sistema metrológico, en el período paleobabilónico reflejan ésto:

$$
\begin{aligned}
& 30 \text { šuši = } 1 \text { kǔs̆ } \\
& 12 \text { kǔš }=1 \text { GAR } \\
& 60 \text { GAR }=1 \text { US }
\end{aligned}
$$

,al kǔs (codo) se le asigna un valor aproximado de $1 / 2$ metro (medida aproximada de la distancia del codo a la mano en una persona). Así GAR, que es la unidad fundamental de longitud, es 6 metros. En área la unidad es-

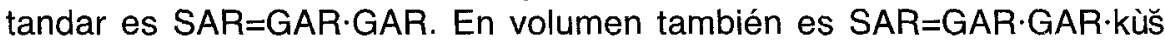
$=6 \cdot 6 \cdot 1 / 2=18 \mathrm{~m}^{3}$.

La costumbre es escribir en el sistema metrológico sexagesimal en sentido ordenado decreciente, expresiones como: 3 US 13 GAR dan origen a expresiones sexagesimales como IIK<Ill que inician la notación Posicional.

\section{La coma}

En principio, un número sexagesimal posicional tiene valor decimal, en el orden de la última cifra, d, según la fórmula:

$$
a, b, c, d=a \cdot 60^{3}+b \cdot 60^{2}+c \cdot 60+d
$$

de este modo 3,13 tiene valor decimal (en el orden del 13):

$3,13=3 \cdot 60+13=180+13=193$.

6 Thureau-Dangin. SHSS. Pág. 110. «La medida natural de peso parece haber sido la que constituye la carga normal para un hombre. Aparentemente, la necesidad de una unidad de peso más pequeña sólo se hizo sentir cuando los metales fueron de uso general y se convirtieron en un articulo de comercio... Si se asume una ratio sexagesimal entre la "carga" (ó sea el talento) y el ma-na, es sin duda debida al hecho de que el número 60 ya era la base de numeración». 
Por otro lado también existen signos específicos para determinadas fracciones:

$$
\text { I es } 1 / 2 \text { iff es } 1 / 3 \text { fff es } 2 / 3
$$

y mediante el uso de frases, como igi $x$ gal que expresa la fracción 1/x, finalmente se expresa cualquier fracción. Pero las fracciones no forman parte del Sistema de Cálculo. El uso de fracciones se limita a la definición de valores reales en datos y resultados. En el Sistema de Cálculo, al realizar operaciones, un número como $31 / 2$ se representa III $\ll<$, ó sea 3,30 . Esto parece contradecir la fórmula (1) pues en ella: $3,30=3 \cdot 60+30=$ 210. Aquí aparece una primera característica del sistema: la no utilización de la coma para separar parte entera y parte fraccionaria. En la notación de Neugebauer ${ }^{7}$ tal función la cumple el punto y coma y $31 / 2$ es $3 ; 30$ mientras que 210 es 3,30 . Pero en el Sistema de Cálculo no existe tal signo y tanto 210 como $31 / 2$ se representan igualmente por 3,30. La diferencia reside en el orden o unidades en que ocurren:

3,30 minutos son $210=3,30$ minutos.

$31 / 2$ minutos son $210=3,30$ segundos. Un orden o unidad metrológica inferior.

Otra característica del sistema es la ausencia de un signo para el cero final. En éste tema es necesario distinguir entre el cero final y el cero intermedio. El tema del cero final está relacionado con la coma, mientras que el cero intermedio sí se llega a expresar ${ }^{8}$.

\section{El cero intermedio}

El cero intermedio es muy improbable en una base tan amplia como es 60 , lo que hace que ocurra en muy pocos textos. En ellos algunas veces no se expresa y su ocurrencia es necesario reconocerla por el contexto ${ }^{9}$. En

7 En el sistema de Neugebauer

$$
a, b, c ; x, y=a \cdot 60^{2}+b \cdot 60+c+\frac{x}{60}+\frac{y}{60^{2}}
$$

8 FRiberg. Pág. 536.

9 Ocurre en BM 85200 \#29 (capítulo de Algebra, Sistemas) y no se expresa. Es reconocible por el contexto de operaciones y resultados; como en « 1,20 y 40 suma y $1,40,20$ es el resultado" en la suma $1,0,20+40,0=1,40,20$. 
otros textos sí se expresa aunque, en general, no de forma plenamente unificada. Según Friberg (op. cit., pág. 536), se expresa el cero intermedio, "a menudo pero ciertamente no siempre" mediante un espacio en blanco:

como en I < <Ill para $1,0,23=1 \cdot 60^{2}+0 \cdot 60+23=3623$.

Pero otras veces se dejan espacios en blanco entre dos cfiras y ello no significa un cero intermedio. En Plimpton 322, la tabla de triples pitagóricos, un número como:

$\ll$ Il es 22,1 y el espacio en blanco se deja para distinguir de $\ll$ lll que es 23.

Los textos-problema de Susa TMS text 24 y TMS text 12 del período Paleobabilónico son, según Nemet-Nejat ${ }^{10}$, el primer uso de un signo, el signo gam 1, para expresar el cero intermedio. Tampoco se usa sistemáticamente pues a veces también se usa para separar cifras. La frase de Friberg (op. cit., pág. 536) en el tema del cero intermedio en el período paleobabilónico es «uso no sistemático». El uso de un signo para el cero intermedio se hace sistemático en la época Seleucida con otro signo, el signo $\}$.

\section{El cero final}

El tema del cero final, es radicalmente distinto. Nunca, como con la coma, se considera necesario crear un signo específico para el cero final. El número decimal 60 se expresaría en la notación de Neugebauer 1,0 y en el sistema Paleobabilónico 1 pues no existe el cero final. La ausencia de cero final sube el orden: 60 minutos son 1,0 minutos (notación de Neugebauer); también 1 hora (notación paleobabilónica). El número se expresa por tanto desde el primer orden en el que ocurre, desde el orden en el que es mayor de cero.

Esta peculiaridad del sistema sin cero final ni coma, flotante en el sistema metrológico o en el orden, produce en los textos matemáticos paleobabilónicos frases chocantes, en suma y resta, como: « 1 y 30 suma y 1,30 tú ves", donde la suma se produce en órdenes diferentes, como en $1 \mathrm{mi}-$ nuto y 30 segundos; en la notación de Neugebauer $1,0+30=1,30$. Tam-

$10 \quad$ Nemet-Nejat. CMTREM. Pág. 37. 
bién «30 en 1 resta y 30 tú ves», en la resta $1,0-30=30$.

Veamos el comportamiento del sistema en la multiplicación. Tenemos lo que ocurre en nuestro sistema decimal, también posicional, al eliminar el cero final y coma: Supongamos una multiplicación como

$$
248 \cdot 124=30752
$$

Si ahora incluimos cero final y coma, vermos que ésta multiplicación realiza infinitas multiplicaciones sin más que corregir el orden del resultado, contando el número de ceros finales $(+n)$ y decimales bajo la coma $(-m)$.

(+1) $2480 \cdot 124=307520=30752 \cdot 10$

(+2) $24800 \cdot 124=3075200=30752 \cdot 10^{2}$ etc.

(-1) $\quad 24,8 \cdot 124=3075,2=30752 / 10$

(-2) $2,48 \cdot 124=307,52=30752 / 10^{2}$ etc.

Una multiplicación Paleobabilónica es una multiplicación como (1), capaz de realizar infinitas multiplicaciones, dicho de otra manera, que realiza la multiplicación en todo orden.

Las Tablas de Multiplicar son creadas en ésta época y una línea de una tabla de multiplicar Paleobabilónica, como "2 veces 4 es 8 ", es ajena e independiente al orden del 4 . El resultado cierto es 8 en cualquier orden, sea 4 (en notación decimal) unidad, decena, centena..., décima, centésima... La multiplicación de dos números $n$ y $m$ es la suma repetida $n$ veces del número $\mathrm{m}$ y por tanto el resultado se produce en el orden del número $\mathrm{m}, \mathrm{y}$, al superar 60, en el(los) orden(es) siguiente(s). El sistema es coherente con ésta capacidad de actuar en todo orden de la tabla de multiplicar.

Más adelante se verá que la división remite a las Tablas de Recíprocos que, como las de multiplicar, son independientes del orden; válidas en cualquier orden. También remite la división a la multiplicación y por tanto también multiplica en modo independiente del orden.

Dado que la notación de Neugebauer, como la nuestra, incluye el orden pues tiene cero final y coma (;) expresa el Valor Real del número, en tanto que el sistema de cálculo Paleobabilónico, que no tiene cero final ni coma, expresa el Valor Flotante (en el sistema de orden) del número. El valor real es proporcional al valor flotante:

Valor real $=$ Valor flotante $\cdot 60^{n}$ por la ausencia de cero final. ej. $1,0=$ 1.60. $1,0,0=1 \cdot 60^{2}$.

Valor real $=$ Valor flotante $/ 60^{\text {n }}$ por la ausencia de comal. ej. $31 / 2=$ 


\section{$3,30 / 60$.}

\section{LAS TABLAS COMBINADAS}

«Los textos Sumerios más antiguos, de donde el sistema sumerio de notación fue deducido, dotando del tiempo de Shulgi, fueron tablas de recíprocos y tablas de multiplicar. Estas últimas aparecen simples o en combinación»" ${ }^{11}$.

\section{La multiplicación}

La Tabla de Multiplicar es una creación típicamente Paleobabilónica. Una tabla de multiplicar simple es una tabla de multiplicar en un número principal $c$.

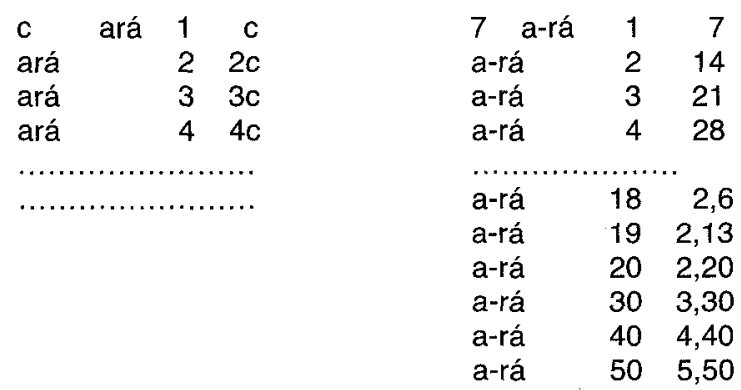

ará se traduce «veces». Tiene 23 líneas, para la multiplicación del número principal c sucesivamente por 1 a 20 y 30, 40, 50. Dos columnas: 1 , $2,3,4, \ldots$ es una progresión de diferencia +1 y la segunda $7,14,21, \ldots$ de diferencia +7 .

Una Tabla de Multiplicar Combinada tiene varias (hasta 40) tablas de multiplicar simples. Ist A 20 + VAT 9734 es una Tabla Combinada. Tiene 39 números principales (falta 2,24); una tabla de cuadrados y otra de recíprocos. La copia esquemática es:

11 Van der Waerden. SA. Pág. 42. 

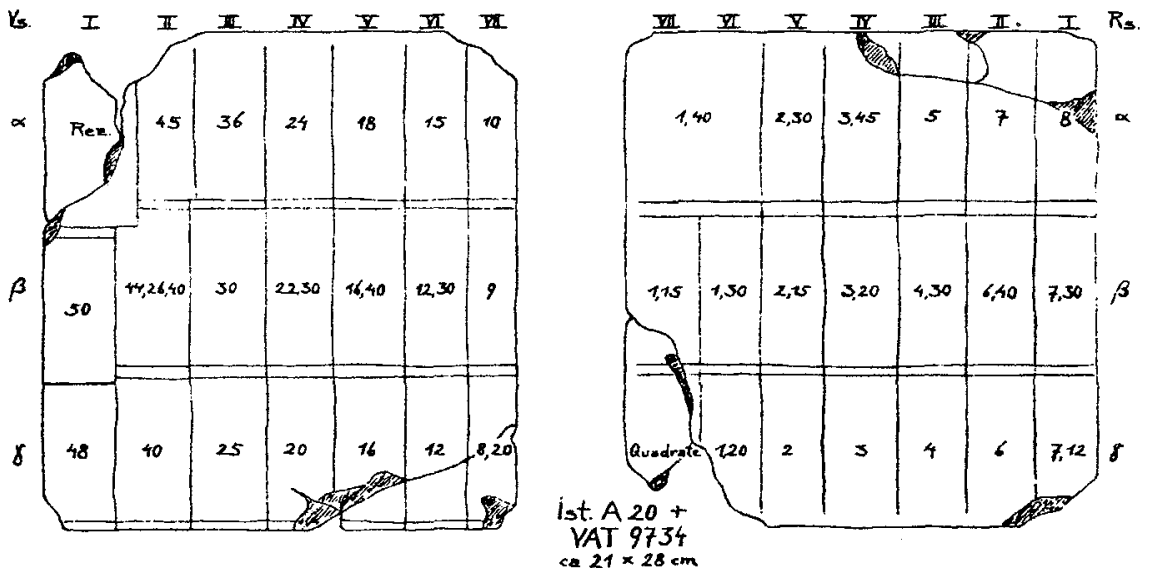

Veamos el fragmento inferior de la tabla del 1,15. Además tiene en las líneas finales el cuadrado de $1,15(1,33,45)$, la raiz cuadrada de $1,33,45$ $(1,15)$, el recíproco de $1,15(48)$ y el recíproco de $48(1,15)$.

\begin{tabular}{|c|c|}
\hline 19 & 23,45 \\
\hline 20 & 25,0 \\
\hline 30 & 37,30 \\
\hline 40 & 50,0 \\
\hline 50 & $1,2,30$ \\
\hline 1,15 a-rá & 1,15 \\
\hline 1,33, & 5 \\
\hline $1,33, \quad[4$ & ] 5 \\
\hline $\begin{array}{r}\text {-e 1,15 } \\
\text { iqi } 1,15\end{array}$ & $\begin{array}{l}{[?]} \\
{[418}\end{array}$ \\
\hline igi 48 [?] & 1,15 \\
\hline
\end{tabular}
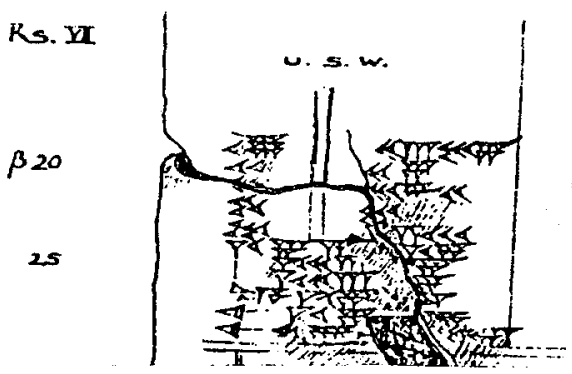

donde $19 \cdot 1,15=19 \cdot 75=1425=23 \cdot 60+45=23,45$ etc.

Para realizar una multiplicación que no figura en la tabla, como $1,15 \cdot 23$, se debería utilizar la propiedad distributiva: $1,15 \cdot 23=1,15 \cdot(20+3)=1,15 \cdot 20$ $+1,15 \cdot 3$. Para obtener resultado correcto, la multiplicación debe ser posicional y distributiva. Como ejemplo la multiplicación $36,23 \cdot 11,3$ con la tabla combinada. 


\begin{tabular}{|c|c|c|c|c|c|c|c|c|c|}
\hline & orden & II & 1 & 0 & & III & II & 1 & 0 \\
\hline $\begin{array}{l}23 \cdot 3 \\
36 \cdot 3 \\
\\
23 \cdot 11 \\
36 \cdot 11\end{array}$ & & & $\begin{array}{r}36 \cdot 3 \\
3 \cdot 11 \\
20 \cdot 11\end{array}$ & $\begin{array}{r}3 \\
20\end{array}$ & $\begin{array}{l}\bar{y} \\
= \\
= \\
= \\
=\end{array}$ & 6 & $\begin{array}{r}3 \\
36\end{array}$ & $\begin{array}{r}1 \\
48 \\
33 \\
40\end{array}$ & 9 \\
\hline 36.11 & & 3.1 & & & $=$ & 6 & 42 & 2 & 9 \\
\hline
\end{tabular}

Como la multiplicación es flotante en el orden, la multiplicación anterior realiza infinitas, en todo orden, multiplicaciones reales, por ejemplo en la notación de Neugebauer:

$36 ; 23 \cdot 0 ; 11,3=6 ; 42,2,9=6,42,2,9 / 60^{3}$.

También:

$36,23,0 \cdot 0 ; 11,3=6,42,2 ; 9=6,42,2,9 / 60$ etc.

El valor real del resultado es proporcional al valor flotante resultado $6,42,2,9$. En el primer caso $\left(/ 60^{3}\right)$ supone tres desplazamientos hacia el orden inferior. En el segundo (/60) un desplazamiento al orden inferior.

En los textos matemáticos, la descripción textual de la multiplicación utiliza frecuentemente el vervo ǐsum = tener.

$$
x \text { a-na y } i \text {-ši } x \cdot \text { y ta-mar (2 a } 3 \text { tiene. } 6 \text { tu ves). }
$$

Después del resultado es frecuente, en todas las operaciones, el verbo ta-mar (amarum = ver), pero también encontramos, ocasionalmente, elum $=$ subir, nadanum $=$ dar.

«Frecuentemente, varias de éstas pequeñas tablas se combinan con tablas de reciprocos y de cuadrados, hasta formar una gran tabla combinada. Antes que podamos entender la organización de tal tabla combinada, debemos mirar las tablas de recíprocos» ${ }^{12}$.

\section{La división}

El recíproco de b es $1 / \mathrm{b}$. Su producto es 1 . La tabla estandar de recíproco es:

12 Van der Waerden. SA. Pág. 42. 


\begin{tabular}{rl|ll|ll}
$\ll 2$ & 30 & 16 & 3,45 & 45 & 1,20 \\
3 & 20 & 18 & 3,20 & 48 & 1,15 \\
4 & 15 & 20 & 3 & 50 & 1,12 \\
5 & 12 & 24 & 2,30 & 54 & $1,6,40$ \\
6 & 10 & 25 & 2,24 & 1 & 1 \\
8 & 7,30 & 27 & $2,13,20$ & 1,4 & 56,15 \\
9 & 6,40 & 30 & 2 & 1,12 & 50 \\
10 & 6 & 32 & $1,52,30$ & 1,15 & 48 \\
12 & 5 & 36 & 1,40 & 1,20 & 45 \\
15 & 4 & 40 & 1,30 & 1,21 & $44,26,40 » 13$
\end{tabular}

En ésta tabla de recíprocos básica tenemos, en la notación de Neugebauer:

$$
1 / 2=0 ; 30,1 / 3=0 ; 20,1 / 4=0 ; 15,1 / 5=0 ; 12 \text {, etc } .
$$

Dado el carácter flotante del sistema, también

$1,0 / 2=30$ y $1,0,0 / 2=30,0$ etc... Es decir, la Tabla de Recíprocos es en todo orden. Veamos la tabla de Ist A 20+VAT9734, muy deteriorada.

$\begin{array}{llll}\text { igi } & 9 & \text { gál-bi } & {[6,40]} \\ \text { igi } & 10 & \text { gál-bi } & {[6]} \\ \text { igi } & 12 & \text { gál-bi } & {[5]} \\ \text { igi } & 15 & \text { gál-bi } & {[4]} \\ \text { igi } & 16 & \text { gál-bi } & {[3,4] 5} \\ \text { igi } & 18 & \text { gál-bi } & {[3,20]} \\ \text { igi } & 20 & \text { gál-bi } & {[3]} \\ \text { igi } & 2 ? & \text { gál-bi } & {[?]} \\ \text { igi } & 24 & \text { gál-bi } & 2,[30] \\ \text { igi } & 27 & \text { gál-bi } & 2,[13,20] \\ \text { igi } & 30 & \text { gál-bi } & 2 \\ \text { igi } & 32 & \text { gál-bi } & {[1,52], 30} \\ \text { igi } & 36 & \text { gál-bi } & {[1,40]} \\ \text { igi } & 40 & \text { gál-bi } & {[1,30]} \\ \text { igi } & 45 & \text { gál-bi } & {[1,20]} \\ \text { igi } & 48 & \text { gál-bi } & {[1,15]} \\ \text { igi } & 50 & \text { gál-bi } & {[1,1] 2} \\ \text { igi } & 54 & \text { gál-bi } & 1,6,40 \\ \text { igi } & 1 & \text { gál-bi } & 1 \\ \text { igi } & 1,4 & \text { gál-bi } & 56,1[5] \\ \text { igi } & 1,21 & \text { gál-bi } & {[4] 4,26,[40]}\end{array}$

igi 9 gál-bi $[6,40]$

igi 10 gál-bi [6]

gi 12 gá-bi [5]

igi 15 gál-bi $[4]$

igi 16 gál-bi $[3,4] 5$

igi 18 gál-bi $[3,20]$

gi gál-bi [3]

gi 2 ? gál-bi [?]

gi 24 gál-bi $2,[30]$

gi 27 gál-bi 2,[13,20]

igi 30 gál-bi

gi 32 gál-bi [1,52],30

igi 36 gál-bi $[1,40]$

igi 45 gál-bi $[1,20]$

igi 48 gál-bi $[1,15]$

igi 50 gál-bi $[1,1] 2$

igi 54 gál-bi $1,6,40$

igi 1 gál-bi 1

igi 1,21 gál-bi [4]4,26,[40]

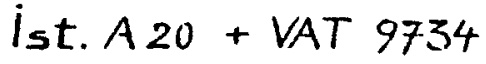

Ys. I

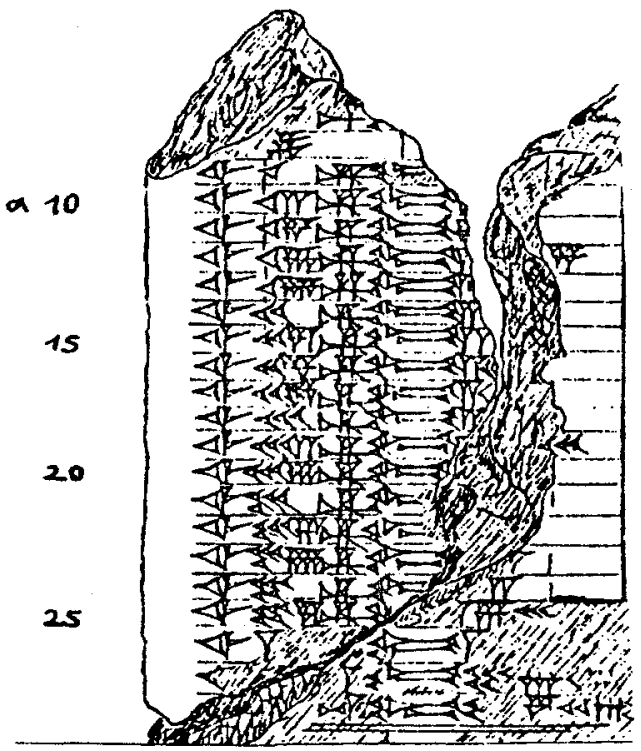

13 Van der Waerden. SA. Pág. 43. 
Como ejemplo, el recíproco de 1,15 es $48: 1,15 \cdot 48=3600=1,00$ (en la transcripción de Neugebauer) $=1$ en Babilonio antiguo pues no utiliza el cero final.

Un modo de generar nuevas tablas de recíprocos es a partir del hecho de que si tenemos dos recíprocos $x$ e $y$, que por tanto cumplen la fórmula $x \cdot y=1$, también se cumple que $2 x \cdot y / 2=1$. Por tanto doble y mitad también son recíprocos. Asi se hace en $\mathrm{BM} 80150$ a partir de los recíprocos 2,5 y 28,48 duplicando el primero y dividiendo a la mitad el segundo. BM 80150 es una tabla de multiplicar combinada con 18 números principales. Tiene también recíprocos.

$\begin{array}{rrrrr}2 & 5 & 28 & 48 & \text { igi-bi } \\ 4 & 10 & 14 & 24 & \text { igi-bi } \\ 8 & 20 & 7 & 12 & \text { igi-bi } \\ 16 & 40 & 3 & 36 & \text { igi-bi } \\ & \ldots . \text {.etc..... } & & & \end{array}$

"para escribir $3 / 8$ en forma sexagesimal, primero localizamos $1 / 8=0 ; 7,30$ en la tabla de reciprocos y después el resultado de ésto, en la tabla de multiplicar por $3 \cdot 0 ; 22,30$. Los textos matemáticos confirman plenamente ésta interpretación. Cuando una división a:b se lleva a cabo en éstos textos, se dice (no en términos de fórmula general, sino por los números especificados): calcula el recíproco $1 / b$ y multiplica por a". ${ }^{14}$.

La división $\mathrm{a} / \mathrm{b}$ tiene dos pasos:

1) Ver en la tabla el reciproco de $b$ que es $1 / b$

2) Multiplicar a por $1 / b$

$$
\text { ej: } \frac{5}{4}=5 \cdot \frac{1}{4}=5 \cdot 0 ; 15=1 ; 15
$$

La divisón se realiza con el procedimiento: «a/b» es «a» veces « $1 / \mathrm{b}$ ». En los textos-problema se refleja ésta manera de dividir:

1) El recíproco se expresa frecuentemente con la forma «igi... gál». igi $=$ ojo, lo que se ve, enfrente gál=ser...:

igi $x$ gál $1 / x$ ta-mar. (enfrente de 10 está. 6 tu ves).

2) La multiplicación de a por $1 / b$ se expresa según la forma de la multiplicación, frecuentemente išum (tener).

i4 Van der Waerden. SA. Pág. 43. 
En suma, tenemos una división $(5 / 4=1 ; 15)$. en dos pasos:

igi 4 gál 15 ta-mar 15 a-na 5 i-ši 115 ta-mar enfrente de 4 está, 15 tú ves. 15 a 5 tiene 1,15 tú ves.

"Aparece, por tanto que las tablas de calcular sumerias estaban organizadas de manera muy útil. Un uso sistemático de las ventajas de la notación posicional evita todo problema con fracciones; las cuatro operaciones racionales se pueden llevar a cabo rápidamente». (Waerden, op. cit., pág. 44.)

Ocasionalmente, pocas veces, se hace la división sin tabla de recíprocos, con el procedimiento directo: «¿cuál es el número $x$ que multiplicado por $b$ da $a$ ? », en la división $a / b=x$. El problema Strssbrg \#1 en el capítulo Algebra-Sistemas tiene una división planteada así.

\section{Cuadrados}

Los textos matemáticos, para describir la operación de elevar al cuadrado, utilizan $K I L$, kalum $=$ retener,... :

$$
x K I L-K I L \mathrm{x}^{2} \text { ta-mar. }
$$

La forma duplicada es en sumerio un modo, para objetos animados, del plural (kur=montaña; kur-kur=montañas) ${ }^{15}$.

Sin embargo las tablas de cuadrados utilizan frecuentemente a-rá (veces). La Tabla Combinada Ist A 20 + VAT 9734 tiene una tabla de cuadrados:

\begin{tabular}{|c|c|c|}
\hline 1 a-rá & [1] & 1 \\
\hline 2 & 2 & [4] \\
\hline 3 & 3 & [9] \\
\hline 4 & 4 & $1[6]$ \\
\hline 5 & 5 & [25] \\
\hline 6 & 6 & $3[6]$ \\
\hline 7 & 7 & 49 \\
\hline 8 & 8 & 1,4 \\
\hline 9 & 9 & 1,21 \\
\hline 10 & 10 & 1,40 \\
\hline 11 & 11 & 2,1 \\
\hline 12 & 12 & $2,[24]$ \\
\hline 13 & 13 & $2,[49]$ \\
\hline 14 & $1[4]$ & {$[3,16]$} \\
\hline 15 & $1[5]$ & {$[3,45]$} \\
\hline
\end{tabular}

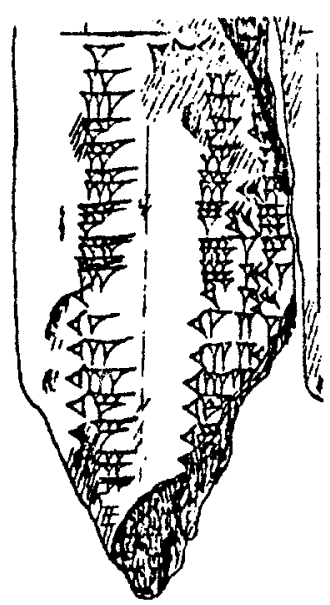

15 HAYES J.L. «A Manual of Sumerian Grammar and Texts». Undena 1990. 


\section{MATEMÁTICAS DE APLICACIÓN}

Clasificando los textos-problema según realicen o no explicación de la resolución del ejercicio que proponen, tenemos dos tipos de textos:

A) Los textos-problema-solución, que no explican el proceso de resolución, tienen el esquema Enunciado-Pregunta-Solución. Un ejemplo es el problema 1 de YBC 4666, una tablilla con una colección de 23 problemassolución sobre la construcción de pequeños canales

YBC 4666 \#1: «Un Canal pequeño. 5 US̆ es su longitud 2 kùs̆ el ancho 1 kùs̆ su profundidad. 1/3 SAR el volumen de la asignación de un trabajador. 1 ban de grano el salario de un trabajador. ¿Cuál es el área el volumen los trabajadores y el total de salarios en grano? 1 ubu es el área 1 ubu el volumen 230 los trabajadores 5 gur el total de grano" ${ }^{16}$.

Adicionalmente, éste tipo de ejercicios tienen enunciado y solución preciso en valores y unidades empleadas.

B) Los textos-problema-procedimiento explican las operaciones necesarias para encontrar la solución. Tienen el esquema Enunciado-PreguntaProceso de Resolución-Solución. El proceso de resolución, generalmente se inicia con za-e (tú) y termina con ki-a-am nepéšum (Así es el procedimiento ${ }^{17}$ ) y es, el proceso de resolución, una descripción encadenada de operaciones con valores numéricos en frases como ...suma 10 y 2 y 12 es el resultado. multiplica 12 por 2 la altura y 24 el resultado es el volumen... etc.

Estos textos, a diferencia de los anteriores, suelen ser menos cuidadosos en la concreción de valores y unidades tanto en el enunciado como en la solución.

La reiterada actividad en éstos textos, en torno a la misma fórmula, dando en un ejercicio unos datos y pidiendo el que falta, $y$ en los siguientes pidiendo otro y dando los demás, en todas las maneras posibles, indica que eran conscientes de la existencia de una relación fija entre las variables, de la existencia de lo que hoy denominamos como fórmula. A continuación los conceptos y fórmula más frecuentes en «matemáticas de aplicación».

\footnotetext{
16 El cálculo de área y volumen es:

El área en $S A R=G A R \cdot G A R$. Además 5 US $=5 \cdot 60 \mathrm{GAR}, 1 \mathrm{GAR}=12$ kúš $\cdot 1$ ubu $=50$ SAR.

$A=1 \cdot a=5,0 \cdot 2 / 12=5 \cdot 60 \cdot 2 / 12=50$ SAR $=1 \mathrm{ubu}$.

El volumen en SAR $=$ GAR.GAR $\cdot k u ́ s$.

$V=A \cdot p=50 \cdot 1=50 \mathrm{SAR}=1 \mathrm{ubu}$.

17 nepésŭm del verbo epésŭm = hacer, se traduce en otros contextos como «ritual».
} 


\section{La Asignación}

Se denomina ${ }^{18}$ asignación (éskàr) al volumen de tierra que debe extraer un hombre contratado en un día de trabajo. La fórmula es ampliamente empleada (a través de valores numéricos concretos) en los textos matemáticos:

$$
V=\dot{e} \cdot t \cdot n \quad \begin{aligned}
& \text { é }=\text { éskàr (asignación) } \\
& t=\text { tiempo en días } \\
& n=\text { número de trabajadores }
\end{aligned}
$$

YBC 4662 es una tablilla de una colección de 30 problemas sobre excavaciones. El problema 28 es:

YBC 4662\#28:

“Una excavación. 5 GAR es la longitud $1 \frac{1}{2}$ GAR el ancho $\frac{1}{2}$ GAR la profundidad. 30 trabajadores terminaron el $9^{\circ}$ día. ¿Cuál es la asignación? Tú, en tu procedimiento, longitud y ancho multiplicas y 7,30 es el resultado. 7,30 por la profundidad multiplica y 45 es el resultado. Multiplica 30 , los trabajadores, y 9 días y 4,30 es el resultado. El reciproco de 4,30 es 13,20. Multiplica 13,20 por 45 y la asignación te dá. 10 gin es la asignación».

Comentario: El volumen, longitud por ancho por profundidad, debe expre-

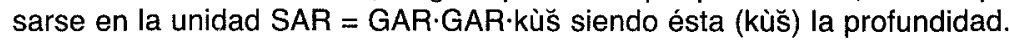

La profundidad $1 / 2$ GAR $=6$ kùs pues $1 \mathrm{GAR}=12$ kùš.

El ancho es $1 \%$ GAR $=1 ; 30$ sexagesimal en la notación de Neugebauer, donde el punto y coma cumple la función de la coma decimal. Este signo (;) no existe en el sistema Paleobabilónico en el cual es 1,30.

El procedimiento es:

$$
\begin{aligned}
& \dot{e}=\frac{V}{n \cdot t}=\frac{1 \cdot a \cdot p}{n \cdot t}=\frac{5 \cdot 1 ; 30 \cdot p}{n \cdot t}=\frac{7 ; 30 \cdot p}{n \cdot t}=\frac{7 ; 30 \cdot 6}{n \cdot t}=\frac{45}{n \cdot t}=\frac{45}{30 \cdot 9}=\frac{45}{4,30}= \\
& =45 \cdot 0 ; 0,13,20=0 ; 10 \text { SAR }=10 \text { gin. }
\end{aligned}
$$

Como 4,30 es la mitad de 9,0 su recíproco es el doble del de 9,0 (6,40 según las tablas) y $2 \cdot 6,40=13,20$ :

$$
\frac{1}{4,30}=13,20=0 ; 0,13,20 \text { en la notación de Neugebauer. }
$$

\footnotetext{
18 Neugebauer. MCT y MKT. Pág. 66.
} 
La longitud que hace 1 hombre en 1 día

Otro concepto que aparece en los textos matemáticos es la longitud que hace, excava, 1 hombre contratado en 1 día $\left(I_{1}\right)$. Las fórmulas ${ }^{19} \mathrm{de}$ proporcionalidad empleadas son:

$$
\begin{aligned}
& I=I_{1} \cdot n \cdot t \\
& e ́=S \cdot I_{1}
\end{aligned}
$$

$I=$ longitud total .

$S=$ Area de la sección frontal (vertical) de la excavación.

YBC 4662 del problema 22 al 28 (problema anterior) tratan de la misma excavación.

YBC 4662\#22.

"Una excavación. 5 GAR es la longitud $1 \frac{1}{2}$ GAR el ancho $1 / 2$ GAR la profundidad. 10 gin la asignación. ¿Cuál es la longitud que realizó un hombre? Tú en tu procedimiento, ancho y profundidad multiplica y 9 es tu resultado. El recíproco de 9 te da 6,40. Multiplica 6,40 por la asignación y $1,6,40$ te dá. 1,6,40 hace un hombre».

Comentario:

$$
I_{1}=\frac{\dot{e}}{s}=\frac{\dot{e}}{a \cdot p}=\frac{\dot{e}}{1 ; 30 \cdot 6}=\frac{\dot{e}}{9}=\dot{e} \cdot 0 ; 6,40=0 ; 10 \cdot 0 ; 6,40=0 ; 1,6,40
$$

Número de ladrillos para cubrir una unidad de área

El número de ladrillos necesarios para cubrir una superficie de 1 SAR área se obtiene mediante la fórmula:

$$
\begin{array}{|l|l}
N \cdot A=1 & \begin{array}{l}
N=\text { número de ladrillos } \\
A
\end{array}=\text { área (en } S A R \text { ) de } 1 \text { ladrillo }
\end{array}
$$

El texto-problema-solución YBC 4607, nombra y describe las dimensiones de cinco tipos de ladrillos; pregunta y dá la solución, para cada uno de los 5 tipos, al número de ladrillos necesarios para cubrir un área de 1 SAR. 


\section{La asignación en Transporte de Ladrillos}

T. Baqir en comentario ${ }^{20}$ al problema IM 54538, cita a Thureau-Dangin: "el transporte de 9,0 (540) ladrillos una distancia de 3 cuerdas ( 3 as-li=30 GAR) es la asignación de un hombre (en 1 día)".

El camino total $C$ del transporte de todos los ladrillos que un trabajador tiene asignados es (número de ladrillos por distancia):

$$
C=9,0 \cdot 30=4,30,0
$$

Este valor aparece en lista de coeficientes como YBC 5022.

Tenemos la fórmula:

$$
n \cdot t \cdot C=d \cdot N
$$

$$
\begin{aligned}
& \mathrm{C}=\text { nazbalum (coeficiente de transporte) } \\
& \mathrm{N}=\text { número de ladrillos } \\
& \mathrm{d}=\text { Distancia }
\end{aligned}
$$

En IM 54538 la asignación es $1,30,0$, por tanto $1 / 3$ de la mencionada $4,30,0$, pues trata de otro tipo (tamaño) de ladrillos. En éste problema se pregunta $\mathrm{n}$, el número de trabajadores necesarios para realizar el transporte, asumiendo $\mathrm{t}=1$ día.

\section{IM 54538:}

"Si alguien te pregunta así: ¿Cuántos trabajadores debería poner para llevar 10 SAR de ladrillos a una distancia de una cuerda durante un día? Tú, en tu procedimiento, pon el coeficiente 1,30 y toma el recíproco de 1,30 tu coeficiente. 40 tú ves. Multiplica 40 por la distancia de una cuerda y 6,40 tú ves. Multiplica 6,40 que tú has visto por 54 que es 10 SAR de tus ladrillos y 6 tú ves. 6 son los hombres que se mantienen transportando todo el día”.

Comentario: La distancia en GAR es $10 \mathrm{GAR}=1$ cuerda.

El número de ladrillos viene expresado como los necesarios para cubrir una superficie de 10 SAR. El texto-problema-solución YBC 4607 da, para 5 tipos de ladrillos (de tamaños) diferentes, el número de ladrillos necesarios para cubrir un área de 1 SAR. Para el tipo 4 es 5,24. Por tanto para 10 SAR de área $\mathrm{N}=10 \cdot 5,24=54,0$ ladrillos.

$$
n=\frac{d \cdot N}{t \cdot C}=\frac{d \cdot N}{1 \cdot 1,30,0}=d \cdot N \cdot 0 ; 0,0,40=N \cdot 10 \cdot 0 ; 0,0,40=N \cdot 0 ; 0,6,40=54,0 \cdot 0 ; 0,6,40=6
$$

\footnotetext{
20 T. BAQUIR. «Some more Mathematical Texts from Tell Harmall». Sumer 7.
} 
Número de ladrillos en una Pila

Se relaciona el número $\mathrm{N}$ de ladrillos y volumen de la Pila mediante la fórmula ${ }^{21}$ de proporcionalidad:

$N=k \cdot V \quad \begin{aligned} & N=\text { número de ladrillos en SAR (1 SAR=720 ladrillos) } \\ & k=\text { coeficiente nalbanum } \\ & V=\text { =volumen de la pila en SAR }\end{aligned}$

YBC 4607, ya mencionado, da el valor de k para 5 tipos (tamaños) de ladrillos. Para el tipo 1, k=7,12. Estos valores también aparecen en listas de coeficientes como YBC 5022. YBC 4708 es una tablilla con 60 problemas-solución sobre éste tema:

YBC 4708\#1:

«Una Pila de Ladrillos. 5 GAR es su longitud $11 / 2$ GAR el ancho $1 / 2$ GAR la altura ¿Cuál es el número de ladrillos? El número de ladrillos es 3 gan y 24 SAR».

Comentario: El volumen, longitud por ancho por profundidad, debe expresarse en al unidad SAR=GAR.GAR-küšs siendo ésta (kùs) la altura.

La altura es $1 / 2$ GAR $=6$ kùš pues 1 Gar $=12$ kùš. Entonces:

$\mathrm{V}=\mathrm{l} \cdot \mathrm{a} \cdot \mathrm{p}=5 \cdot 1 ; 30 \cdot 6=5 \cdot 9=45 \mathrm{SAR}$

$\mathrm{N}=\mathrm{k} \cdot \mathrm{V}=7 ; 12 \cdot 45=5,24 \mathrm{SAR}=324$ (decimal) $\mathrm{SAR}=3$ gan y 24 SAR pues 1 gan es 100 SAR.

Las Matemáticas de aplicación en el período Paleobabilónico utilizaban fórmulas con coeficientes fijos denominados igi-gub (gub=plantar). Entre ellos, nazbalum es el coeficiente para el transporte de ladrillos y nalbanum es el coeficiente para el número de ladrillos en una pila. Estos coeficientes están en razón al tamaño del ladrillo.

La tablilla YBC 5022 (abajo la cara Vs) es una lista de coeficientes fijos utilizados en diversos temas. En las trece primeras líneas, se mencionan los coeficientes nazbalum y nalbanum para seis tipos (tamaños) de ladrillos. Los nombres de los ladrillos son: $\operatorname{sig}_{4}$ (ladrillo), $\operatorname{sig}_{4}$-áb (medio-ladrillo), $\mathrm{sig}_{4}$-al-ùr-ra (iadrillo-codido), $1 \mathrm{kùs} \mathrm{sig}_{4}, 1 / 2 \mathrm{kùs} \mathrm{sig}_{4}$, $1 / 3 \mathrm{kùs} \mathrm{sig}_{4}$. Las cabeceras de las columnas, igi-gub-ba hace referencia a los coeficientes y ša nepištum hace referencia al procedimiento o actividad.

21 Neugebauer. MCT. Pág. 137. 


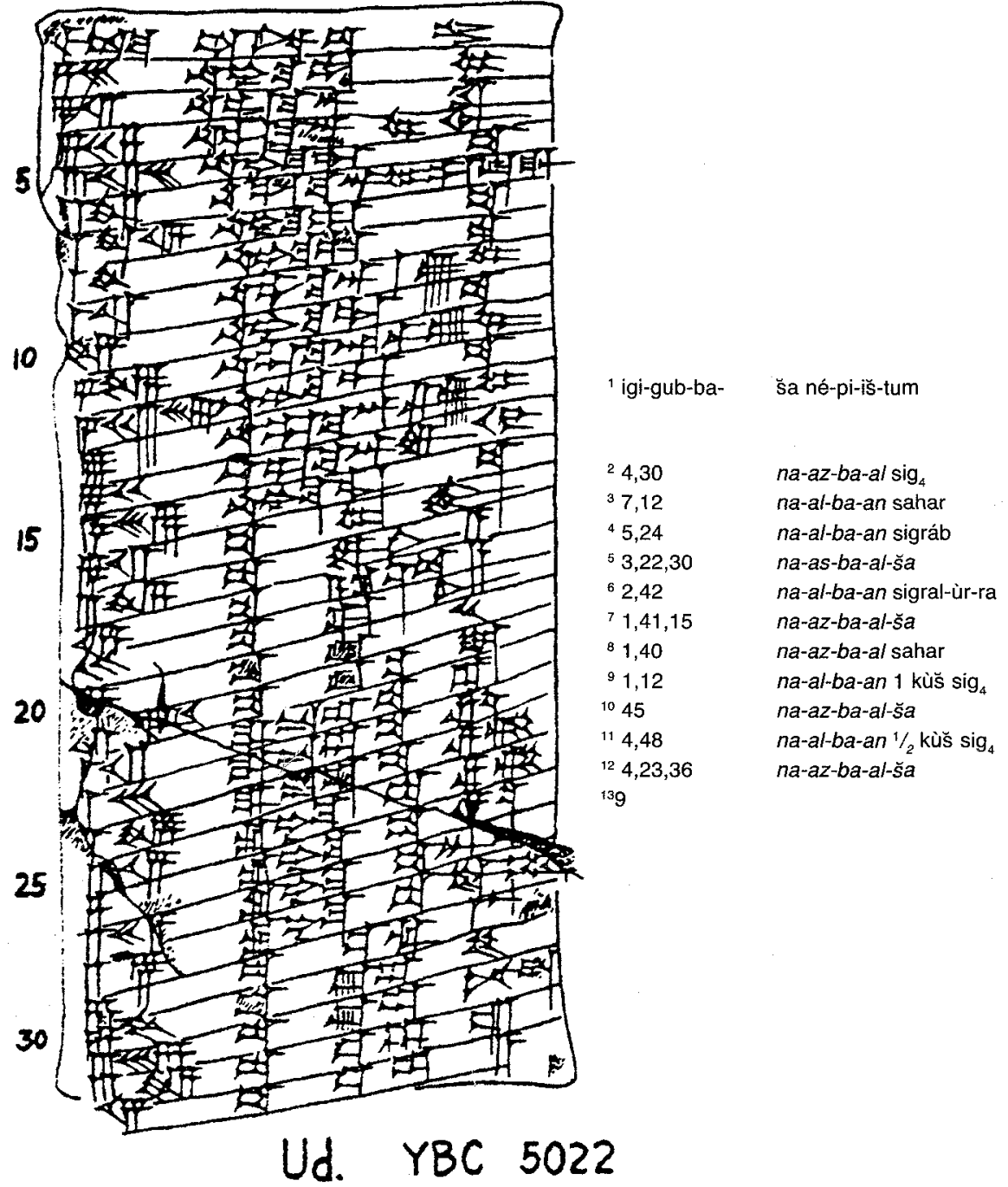

YBC 4708 es una tablilla Paleobabilónica con 60 problemas-solución sobre Pilas de Ladrillos. Debajo la copia de la tablilla y la transcripción y traducción de los 4 primeros problemas. En los cuatro primeros problemas tenemos la misma pila y se pregunta sucesivamente el número de ladrillos (233280), la longitud, el ancho y la altura de la pila. 


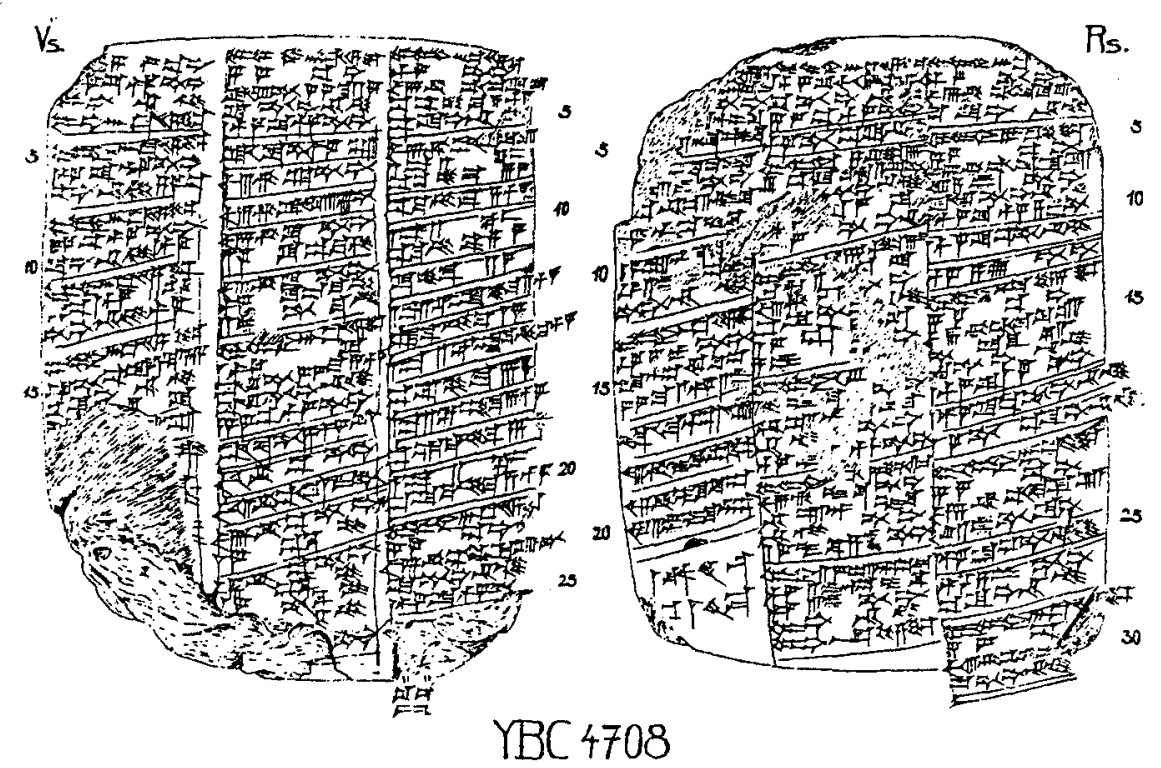

\#1 Ver página 14.

\#2 Pila con 3 gan 24 SAR ladrillos

$11 \frac{2}{2}$ GAR es el ancho $1 / 2$ GAR su altura

¿Cuál es la longitud? 5 GAR es la longitud

\#3 Pila con 3 gan 24 SAR ladrillos

5 GAR es la longitud $1 / 2$ GAR su altura

¿Cual es el ancho? $1 \frac{1}{2}$ GAR

\#4 Pila con 3 gan 24 SAR ladrillos

5 GAR es la longitud $1 \frac{1}{2}$ GAR el ancho

¿Cual es la altura? $1 / 2$ GAR
TRANSKRIPTION

Vs. 1.

$11\left[\mathrm{SIG}_{4} \mathrm{SIG}_{4}\right.$-GI]R 5 GAR us̀-bi

${ }^{2}[11 / 2]$ GAR sag $1 / 2$ GAR sukud-bi

${ }^{3} \mathrm{SIG}_{4}$-bi en-nam

${ }^{4} \mathrm{SIG}_{4}$-bi 3 (gán) gán 24 SAR

$2{ }^{5} \mathrm{SIG}_{4} \mathrm{SIG}_{4}$-GIR 3 (gán) gán 24 SAR

${ }^{6} 1 \frac{1}{2}$ GAR sag $1 / 2$ GAR sukud-bi

7 us̀-bi en-nam 5 GAR us̀

$3{ }^{8} \mathrm{SIG}_{4} \mathrm{SIG}_{4}$-GIR 3 (gán) gán 24 SAR

${ }^{9} 5$ GAR us $1 / 2$ GAR sukud-bi

${ }^{10}$ sag-bi en-nam $1 \frac{1}{2}$ GAR

$4{ }^{11} \mathrm{SIG}_{4} \mathrm{SIG}_{4}-\mathrm{GIR} 3$ (gán) gán $24 \mathrm{SR}$

${ }^{12}$ [5 G]AR uś $1 \frac{1}{2}$ GAR sag

13 sukud-bi en-nam $1 / 2$ GAR

\section{ALGEBRA}

«...los Matemáticos de Babilonia fueron maestros en el arte de manipular expresiones algebraicas elementales pero a menudo complejas...» (Friberg. Pág. 571).

En los siguientes ejemplos vemos procedimientos básicos del Algebra actual:

1) Aplican las reglas de trasposición de términos que empleamos en la actualidad y que resuelven todas las ecuaciones de primer grado: 

Si $A+B=C$ entonces $A=C-B$
Si $A-B=C$ entonces $A=C+B$
Si $A \cdot B=C$ entonces $A=C / B$
Si $A / B=C$ entonces $A=C \cdot B$

2) Fórmulas para resolver ecuaciones de segundo grado. S. Gandz en "The origin and development of cuadratic equations in Babylonian, Greek and early Arabic Algebra" expone los tres tipos en que clasificaban las ecuaciones de $2^{\circ}$ grado y, en cada caso, la fórmula de resolución.

3) Fórmulas para resolver una amplia gama de sistemas. Es el único método que actualmente no se utiliza.

4) Utiliza el método de sustitución en Sistemas (un ejemplo es el ejercicio de ecuación de $2^{\circ}$ grado).

5) Utilizan el método del cambio de variable (ejercicio segundo de Sistemas).

\section{Ecuaciones de primer grado}

IM $53957^{22:}$

Si alguien te pregunta así: Si sumo a los dos tercios de mis dos tercios 100 qa de grano la suma es la cantidad original. ¿Cuál es mi cantidad original? Tú en tu procedimiento multiplica $2 / 3$ por $2 / 3$ y 26,40 tú ves. 26,40 en 1 resta y 33,20 es el resultado. El recíproco de 33,20 rompe y 1,48 tú ves. Multiplica 1,48 por 1,40 y 3 tú ves. 3 es la cantidad original.

Comentario: $x=$ cantidad original. Teniendo en cuenta que $100=1,40$ sexagesimal tenemos la ecuación:

$$
\begin{aligned}
& \frac{2}{3} \cdot \frac{2}{3} x+1,40=x \text { que como } 2 / 3 \cdot 2 / 3=0 ; 26,40 \\
& \text { queda } \\
& 0 ; 26,40 \cdot x+1,40=x \\
& 1,40=x \cdot(1-0 ; 26,40)=x \cdot 0 ; 33,20 \\
& x=\frac{1,40}{0 ; 33,20}=1,40 \cdot 1 ; 48=3,0
\end{aligned}
$$

22 T. BAQIR «Some more Mathematical Texts from Tell Harmall». 


\section{Ecuaciones de segundo grado}

Este ejemplo resuelve la ecuación de segundo grado

$$
a \cdot x^{2}-2 \cdot b \cdot x=c
$$

mediante la fórmula ${ }^{23}$ :

$$
x=\frac{b+\sqrt{b^{2}+a c}}{a}
$$

, la ecuación se obtiene por sustitución de "y» en un sistema. Además utiliza la fórmula del cuadrado de una resta: $(a-b)^{2}=a^{2}+b^{2}-2 \cdot a \cdot b$

\section{Strssbr 363 \#1:}

El área de 2 cuadrados suma 16,40. El lado del cuadrado menor es 2/3 del lado del cuadrado mayor menos 10. ¿Cuáles son los lados? Tú en tu procedimiento 10 al cuadrado 1,40 da. 1,40 en 16,40 resta y 15 ha dado. 1 al cuadrado 1 da. 40 al cuadrado 26,40. 1 y 26,40 suma 1,26,40 da. Multiplica 1,26,40 por 15 y 21,40 da. 40 al cuadrado 26,40. 1 y 26,40 suma 1,26,40 da. Multiplica 1,26,40 por 15 y 21,40 da. 40 por 100 multiplica y 6,40 da. 6,40 al cuadrado $44,26,40$ da. 44,26,40 a 21,40 suma $22,44,26,40$ ha dado. $22,44,46,40$ tiene raiz 36,40. 6,40 que has retenido a 36,40 suma 43,20 da. ¿Cuál es el que a 1,26,40 multiplicó y 43,20 ha dado? 30 pon. 30 por 1 multiplica 30 es el lado mayor. 30 por 40 multiplica y 20 da. 10 en 20 resta y 10 es lado menor.

Comentario

$$
\begin{array}{l|l}
\text { Sistema } & \begin{array}{l}
x^{2}+y^{2}=16,40 \\
\frac{2}{3} \cdot x-10=y
\end{array}
\end{array}
$$

la segunda ecuación (sexagesimal) es $0 ; 40 \cdot x-10=y$

Por Sustitución en la primera ecuación queda: $x^{2}+(0 ; 40 x-10)^{2}=16,40$. Desarrollando por la fórmula $(a-b)^{2}=a^{2}+b^{2}-2 \cdot a \cdot b$

$x^{2}+0 ; 40^{2} \cdot x^{2}+10^{2}-2 \cdot 0 ; 40 \cdot x \cdot 10=16,40$. Como $10^{2}=1,40$ y $0 ; 40^{2}=0 ; 26,40$ $x^{2} \cdot(1+0 ; 26,40)-2 \cdot 0 ; 40 \cdot x \cdot 10=16,40-1,40$

$1 ; 26,40 \cdot x^{2}-2 \cdot 0 ; 40 \cdot 10 \cdot x=16,40-1,40=15,0$ early Arabic Algebra». OSIRIS 3. Pág. 405. 


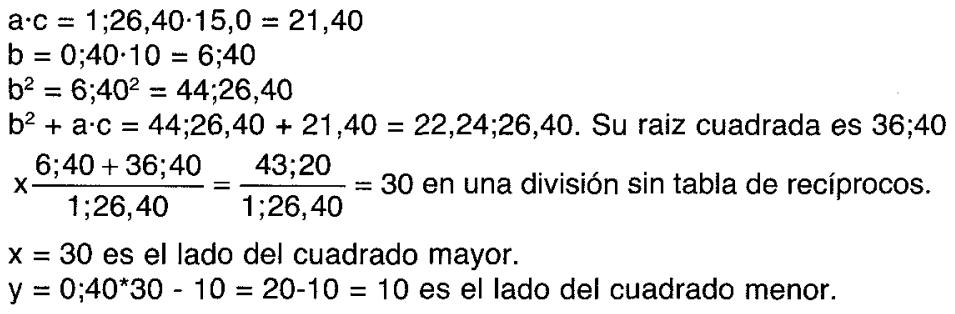

\section{Sistemas}

S. Gandz clasifica los sistemas algebraicos en 6 tipos (B I a B VI). tipo $B$ I sistema fórmula solución

$$
\begin{array}{l|ll}
x+y=a & x=a / 2+w & \text { siendo } w=\sqrt{(a / 2)^{2}-b} \\
x \cdot y=b & y=a / 2-w
\end{array}
$$

tipo B II sistema

$$
\begin{array}{l|ll}
x-y=a & x=w+a / 2 & \text { siendo } w=\sqrt{(a / 2)^{2}+b} \\
x \cdot y=b & y=w-a / 2
\end{array}
$$

La resolución de los sistemas $B$ I y $B$ II implica encontrar el rectángulo de lados $x$ e y conocidos, además del área $b=x \cdot y$, en $B I$ el semiperímetro $\mathrm{a}=\mathrm{x}+\mathrm{y}$; en $\mathrm{B}$ II la diferencia $\mathrm{a}=\mathrm{x}-\mathrm{y}$ lado mayor menos lado menor.

\section{BM 85200\#18:}

Longitud y ancho. La longitud es "igi». El ancho es "igi-bi", su reciproco. Tanto como la suma de «igi» e «igi-bi» es la altura. 30 es el volumen. Tú, el recíproco de 12 mira 5 tú ves. 5 por 30 el volumen multiplica 2,30 tú ves $1 / 2$ a 2,30 rompe. Eleva al cuadrado. 1,33,45 tú ves. 1 en $1,33,45$ resta 33,45 tú ves. 45 la raiz cuadrada, a 1,15 suma y resta. 2 y 30 tú ves. Procedimiento.

Comentario

$$
\begin{aligned}
& I=\text { longitud }=\text { «igi } », \\
& a=\text { ancho }=\text { «igi-bi», su recíproco (i.e. } \mid \cdot a=1 \text { ) } \\
& p=\text { altura }
\end{aligned}
$$

El estandar de unidades es I y a en GAR, $p$ en kùs, Volumen en SAR (SAR $=$ GAR $^{\star} \mathrm{GAR}^{*}$ kùs).

$$
\text { Datos: I * } a=1, \quad \quad 1+a=p, \quad V=30
$$

El cálculo de la altura es evidente y no lo hace.

$V=30=l^{*} a^{*} p=1^{*} p=p, p=30$ kùs 
Homogeniza las unidades: $p=30 / 12=30^{*} 0 ; 5=2 ; 30$ GAR

Tenemos el sistema tipo B I (S. Gadz)

$$
\mid \begin{aligned}
& 1+a=2 ; 30 \\
& 1 * a=1
\end{aligned}
$$

y su fórmula de resolución: $0 ; 45$

$(a / 2)^{2}-b=(2 ; 30 / 2)^{2}-b=(1 ; 15)^{2}-b=1 ; 33,45-1=0 ; 33,45$. Su raiz cuadrada es:

$$
\begin{aligned}
& x=1 ; 15+0 ; 45=2 \\
& y=1 ; 15-0 ; 45=0 ; 30
\end{aligned}
$$

Este problema aplica el método del cambio de variable. Aparece además un número $(2,25)$ con cero intermedio que no se expresa (en vez de $2,0,25)$.

\section{BM 85200 \#29:}

Longitud $y$ ancho. 1,40 es la longitud. 1/7 de la diferencia longitud sobre ancho y 2 kùs es la altura. 3,20 es el volumen. ¿Cual es el ancho y la altura? Tú, 1,40 la longitud por 12 multiplica 20 tú ves. El recíproco de 20 mira 3 tú ves. 3 por 3,20 multiplica 10 tú ves. 10 por 7 multiplica 1,10 tú ves. 1,40 a 1,10 suma 2,50 tú ves. $1 / 2,2,50$ rompe y eleva al cuadrado 2,25 tú ves. En 2,25 resta 1,10 y 50,25 tú ves. 55 la raiz cuadrada a 1,25 suma y resta. 2,20 y 30 el ancho tú ves. $1 / 7$ de 2,20 es 20 la altura. Procedimiento.

Comentario

$$
\begin{aligned}
\text { Datos } \quad \begin{aligned}
l & =1,40 \\
(1,40-\mathrm{a}) / 7+2 \text { kùs } & =p \\
V & =3,20
\end{aligned} \text { por tanto } 7 \mathrm{p}+\mathrm{a}=1,40+14 \text { kùs } \\
\text { El estandar de unidades es I en GAR, a en GAR, } p \text { en kùs, volumen en } \\
\text { SAR (SAR = GAR * GAR * kùs). }
\end{aligned}
$$

Cálculo de altura por ancho. Pone I en kùs (1 GAR = 12 kùs)

$$
a^{*} p=\frac{V}{1}=\frac{3,20}{1,40^{*} 12}=\frac{3,20}{20}=3,20 * 3=10 \mathrm{GAR}^{*} \mathrm{GAR}
$$

Cálculo de $7^{\star} 2$ kùs $=7^{\star} 2 / 12$ GAR $=7^{\star} 2^{\star} 5=7^{\star} 10=1,10$ GAR

$$
1,40+1,10=2,50
$$

Cálculo de $7^{\star} \mathrm{a}^{*} \mathrm{p}=7^{\star} 10=1,10 \mathrm{GAR}^{\star} \mathrm{GAR}$

Tenemos el sistema

$$
\mid \begin{aligned}
& a+7 p=2,50 \\
& a * 7 p=1,10
\end{aligned}
$$


Sistema tipo B I con cambio de variable (S. Gandz).

haciendo el cambio de variable $x=7 p$ queda el sistema

$$
\begin{aligned}
& x+a=2,50 \\
& x * a=1,10
\end{aligned}
$$

sistema tipo B I que resuelve aplicando la fórmula (S. Gandz).

$(2,50 / 2)^{2}-b=(1,25)^{2}-b=2,0,25-1,10=50,25$. Su raiz cuadrada es 55

$x=1,25+55=2,20$

$a=1,25-55=30$

$Y$ deshace el cambio: $p=x / 7=2,20 / 7=20$
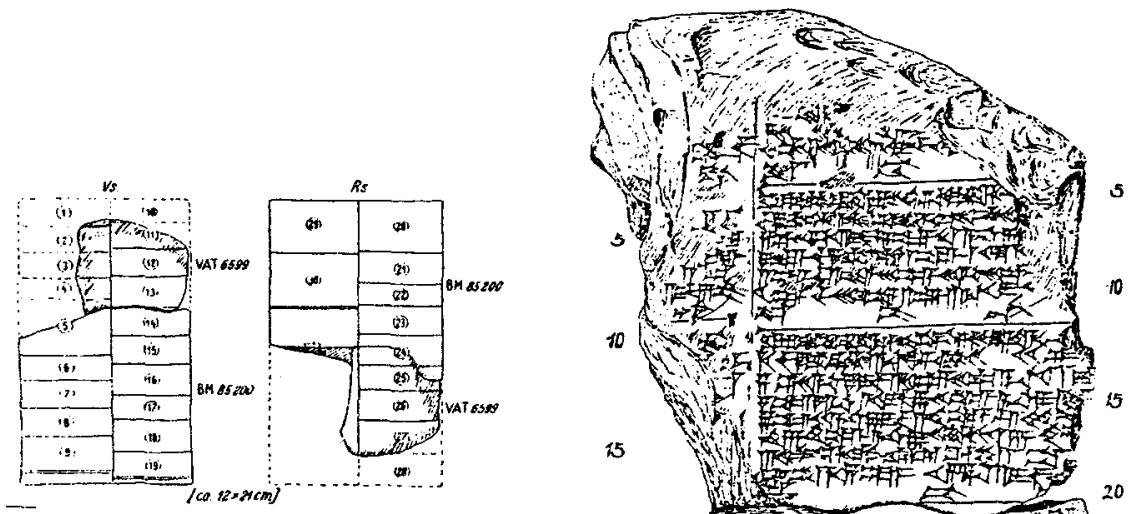

5M $85200+$ VAT 6599

's.




BM 85200 + VAT 6599 es una tablilla Paleobabilónica enteramente dedicada a problemas de Algebra. La reconstrucción de Neugebauer tiene 30 problemas de los que se conservan del 5 (parcialmente) al 27,29 y 30 . En todos los problemas se propone y explica la resolución de sistemas. Los problemas \#18 y \#29 se tratan en las páginas 85 y 86.

\section{GEOMETRÍA}

\section{Cálculos de áreas y volumenes}

BM 85194\#1, utiliza para el volumen de una forma geométrica Irregular (una rampa de acceso a la puerta de una ciudad) una fórmula aproximada de promedios:

Volumen $=$ promedio de los anchos $\cdot$ promedio de las alturas $\cdot$ longitud

$$
v=\frac{1}{2}\left(\frac{b i+b s}{2}+\frac{a s+a i}{2}\right) \frac{a+b}{2} \cdot 1
$$$$
\begin{aligned}
& b s=1 \text { GAR } \\
& b=6 \text { kus } \\
& b i=1 ; 30 \text { GAR } \\
& \text { as }=0 ; 30 \text { GAR } \\
& a=4 \text { kus } \\
& a i=\text { GAR }
\end{aligned}
$$

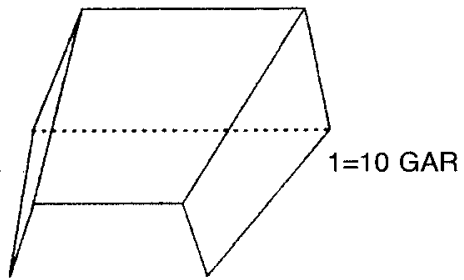

En formas Geométricas Regulares, se obtiene, a final de éste período, fórmula exacta para el Tronco de Piramide Regular de base Cuadrada. Los textos-problema YBC 4607, YBC $5037^{24}$ calculan el volumen de un tronco de pirámide regular de altura $p$, de bases cuadradas con lados superior Is, inferior li, con la fórmula aproximada; promedio de las áreas por altura:

$v=\frac{\mid i^{2}+l s^{2}}{2} \cdot p$

BM 85194\#28 ${ }^{25}$ utiliza la fórmula exacta:

$$
v=\left[\left(\frac{\mathrm{l}+\mathrm{Is}}{2}\right)^{2}+\frac{1}{3} \cdot\left(\frac{\mathrm{li}-\mathrm{ls}}{2}\right)^{2}\right] \cdot \mathrm{p}
$$

Is

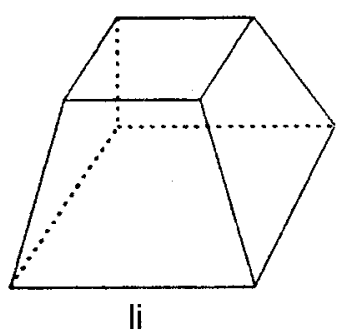

24 Fechados «Babilonio Antiguo». Neugebauer. MCT.

25 Fechado «Período Casita o anterior». Neugebauer. MKT. 
La proporcionalidad. El Teorema de Tales

En textos-problema de Geometría se aplica la proporcionalidad a triángulos rectángulos (Teorema de Tales) y a triángulos isósceles. También se aplica la proporcionalidad en áreas.

Triángulos rectángulos

BM 85210 problemas \#1,\#2,\#3 y \#4 tratan de una pendiente sobre un muro. El Teorema de Tales resuelve los cuatro problemas.

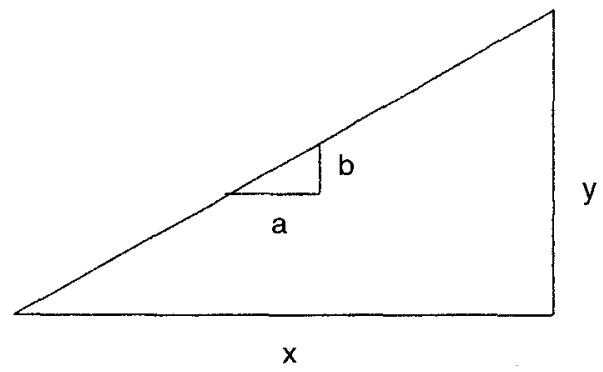

BM 85210 \#1: Una ciudad vi. 3 GAR es la altura del muro $21 / 2$ kùs el paso del escalón 1,40 kùs vertical del escalón. ¿Cuál es la longitud? La ciudad enemiga de Marduk conquistaré. Tù: El recíproco de 1,40 la altura calcula 36 tu ves. 36 por 2,30 multiplica 1,30 tu ves. 1,30 por 3 la altura del muro multiplica 4,30 tu ves. 4,30 es la distancia que pisaste. Procedimiento.

BM 85210 \#2: 2,30 kùs es el paso del escalón de 1,40 vertical 4,30 la distancia. ¿Cuál es la altura del muro? Tu: El recíproco de 1,40 por 2,30 multiplica 1,30 ves. El reciproco de 1,30 calcula 40 tu ves. 40 por 4,30 la distancia multiplica 3 tu ves. 3 GAR es la altura del muro. Procedimiento.

BM 85210 \#3: 3 GAR es la altura del muro 4,30 la distancia 2,30 kùs el paso. ¿Cuál es la vertical? Tu: El reciproco de 4,30 calcula 13,20 tu ves. 13,20 por 2,30 el paso multiplica 33,20 tu ves. 33,20 por 3 la altura del muro multiplica 1,40 tu ves la vertical. Procedimiento.

BM 85210 \#4: 3 GAR es la altura del muro 4,30 la distancia 1,40 la vertical. ¿Cuál es el paso del escalón? Tu: El recíproco de 4,30 la distancia calcula 13,20 tu ves. 13,20 por 3 la altura del muro multiplica 40 tu ves. El recíproco de 40 calcula 1,30 tu ves. 1,30 por 1,40 la vertical multiplica 2,30 tu ves 2,30 kùs es el paso del escalón. Procedimiento. 
Comentario:

$x=4 ; 30 \mathrm{GAR}=$ longitud

Lo pide \#1

$y=3$ GAR altura del muro

Lo pide \#2

$a=2 ; 30$ kus el paso del escalón

Lo pide \#4

$b=1 ; 40$ kus vertical del escalón

Lo pide \#3

$\# 1$

$$
x=\frac{y \cdot a}{b}=\frac{y \cdot a}{1,40}=y \cdot a \cdot 36=y \cdot 2,30 \cdot 36=y \cdot 1,30=3 \cdot 1,30=4,30
$$

\#2

$$
y=\frac{x}{a / b}=\frac{x}{2,30 / 1,40}=\frac{x}{1,30}=x \cdot 40=4,30 \cdot 40=3
$$

\#3

$$
b=\frac{y \cdot a}{x}=\frac{y \cdot a}{4,30}=y \cdot 2,30 \cdot 13,20=y \cdot 33,20=3 \cdot 33,20=1,40
$$

\#4

$$
a=\frac{b}{y / x}=\frac{b}{y / 4,30}=\frac{b}{y \cdot 13,20}=\frac{b}{3 \cdot 13,20}=\frac{b}{40}=1,40 \cdot 1,30=2,30
$$

En la matemática paleobabilónica, la pendiente de una recta se define con el texto:

i-na 1 kùs a sà-gal (en 1 kùs "a» es el «alimento»).

Lá vertical es siempre 1 ( $b=1$ kùs). La horizontal es variable $a=2$ (por ejemplo) kùs según la pendiente a describir. Estos problemas utilizan el Teorema de Tales, para resolver la proporcionalidad entre los valores del escalón de la pendiente $(a, 1)$ y los valores $(x, y)$ del objeto del problema en cuestión. Como $b=1$, la fórmula anterior (1) queda: $a=x / y$. BM 85194 \#17, \#18 y \#28, también BM 85210 \#8 tratan de pendientes definidas y resueltas de éste modo.

YBC 7289 aplica la proporcionalidad entre un cuadrado de lado 1 y otro del lado 30 . Calcula la diagonal del cuadrado de lado 30 a partir de $\sqrt{2}$ la diagonal del cuadrado de lado 1. 
YBC 7289
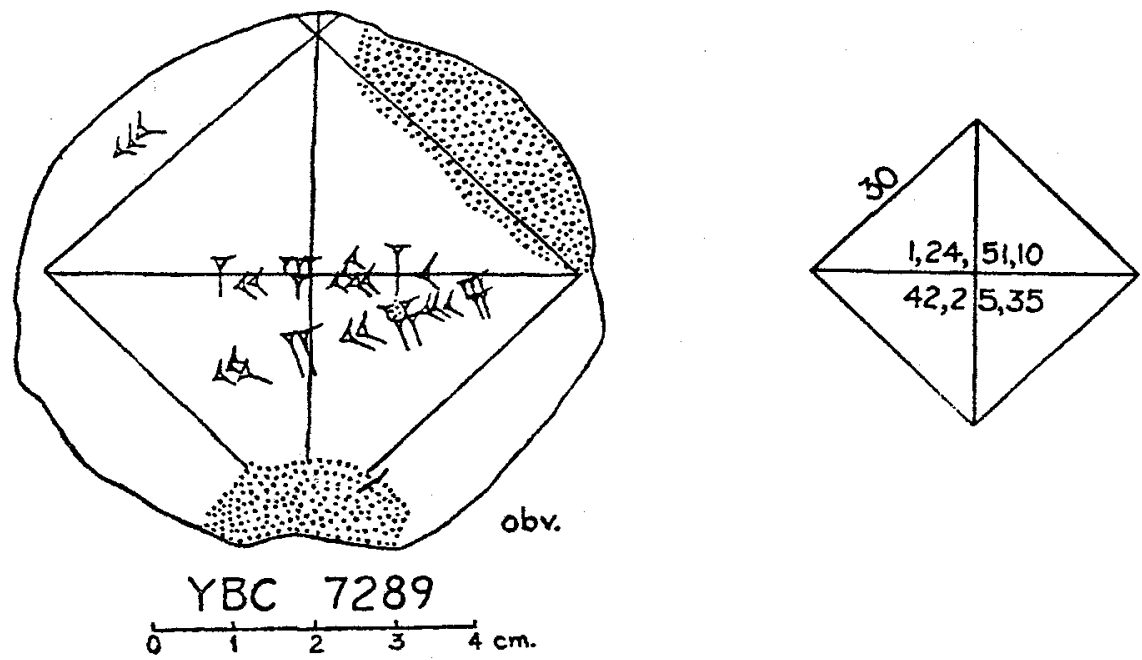

Comentario (Neugebauer MCT). Pg. 43.

«El número 30 indica el lado a del cuadrado y 1,24,51,10 significa: (1) $1,24,51,10$ aproximación de $\sqrt{2}$

por tanto encontramos $d=a \sqrt{2}=42 ; 25,35$ para la diagonal.

El valor (1) para $\sqrt{ } 2$ es muy bueno, como se puede ver en $1,24,51,10^{2}=$ $1,59,59,59,38,1,40$ ”

(En la lista de coeficientes YBC 7243 encontramos)

$1,24,51,10$ «diagonal, raiz cuadrada».

Triángulos isósceles

BM $85194 \# 16$ y \#19, con el tema de un pozo revestido por una corona circular de ladrillos, utilizan el Teorama de Tales para triángulos isósceles:

$$
\frac{\mathrm{R}-\mathrm{r}}{\mathrm{r}}=\frac{\mathrm{as}-\mathrm{ai}}{\mathrm{ai}}
$$
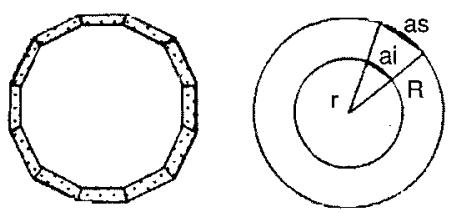
donde: $R=$ Radio exterior

$r=$ radio interior

as = ancho superior del ladrillo

$\mathrm{ai}=$ ancho inferior del ladrillo



Areas

BM 85194 \#26 aplica razón y/x para transformar un rectánculo en un cuadrado:

$$
y^{2}=x \cdot y \cdot \frac{y}{x}
$$

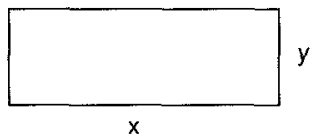

Teorema de Pitágoras

El Teorema de Pitágoras establece que en todo triángulo rectángulo $\mathrm{d}^{2}=\mathrm{u}^{2}+\mathrm{S}^{2}$. El teorema puede ser intuido a partir de la transformación del cuadrado $s^{2}$ en el gnomon que completa el cuadrado $u^{2}$, originando un cuadrado aproximadamente igual a $\mathrm{d}^{2}$.
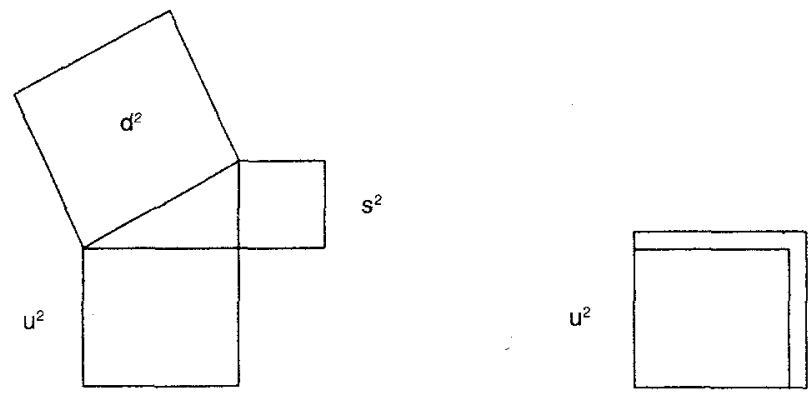

En la Matemática Paleobabilónica el Teorema de Pitágoras se aplica en triángulos rectángulos semejantes al de lados 3,4 y 5 de modo claro. Quizás porque sea una forma segura de tener solución exacta en la raiz cua- 
drada, el hecho es que excepto la tablilla Plimton 322 todos los problemas relativos al teorema de Pitágoras tratan con triángulos rectángulos semejantes a $(3,4,5)$.

BM 85194 \#20 y \#21 son dos problemas sobre una cuerda de una circunferencia que utilizan en su resolución el Teorema de Pitágoras. El triángulo rectángulo que se considera tiene lados $(12,16,20)$, por tanto semejante al triángulo rectángulo $(3,4,5)$. En el problema \#20 se pide la longitud de al cuerda. En el \#21 se pide la profundidad de la cuerda.

\section{BM85194 \#20}

1 es la circunferencia. ¿Cuál es la cuerda a 2 de profundidad? Tú 2 duplica 4 tú ves. 4 en 20 el diámetro resta 16 tú ves. 20 el diámetro al cuadrado 6,40 ves 16 al cuadrado 4,16 tú ves. 4,16 en 6,40 resta 2,24 tú ves. 2,24 ¿cuál es la raiz cuadrada? 12 la raiz

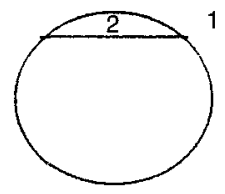
cuadrada es la cuerda. Así es el procedimiento.

Comentario

$\mathrm{L}=$ longitud circunferencia $=1$

$\mathrm{d}=$ diámetro

$a=$ profundidad de la cuerda $=2$

$\mathrm{I}$ = longitud de la cuerda

En un cálculo conocido al nivel de éste problema

$L=3 d=1$ por tanto $d=20$

Tenemos el Teorema de Pitágoras

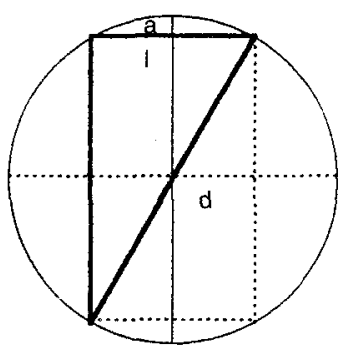

$$
\begin{aligned}
& I=\sqrt{d^{2}-(d-2 a)^{2}} \quad \text { aplicado } a(12,16,20)=4(3,4,5) . \\
& I=\sqrt{20^{2}-(20-2 \cdot 2)^{2}}=\sqrt{6,40-16^{2}}=\sqrt{6,40-4,16}=\sqrt{2,24}=12
\end{aligned}
$$

BM 85196 \#9 es un problema-procedimiento: “Una escalera de longitud 30 apoyada sobre una pared desliza 6. ¿Cuánto se separa de la pared en la parte inferior?..”

La solución que da el texto es

$$
\begin{aligned}
& x=\sqrt{30^{2}-(30-6)^{2}}=\sqrt{15-24^{2}}= \\
& =\sqrt{15-9,36}=\sqrt{5,24=18}
\end{aligned}
$$

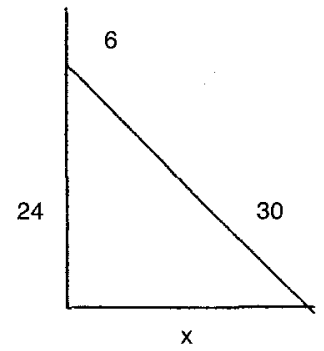

El triángulo rectángulo es por tanto $18,24,30)=6(3,4,5)$ 
Según Friberg otros problemas sobre el Teorema de Pitágoras en triángulos rectángulos semejantes a $(3,4,5)$ son: $\mathrm{Db}_{2} 146$ (publicados en Sumer 18), YBC 8633 (publicado en MCT), TMS text 1 (TMS), IM 55357 (Sumer 6), VAT 7531 (MKT).

\section{Plimpton 322}

Plimpton 322 es una tablilla rota por su zona izquierda. Conserva cuatro columnas de números con cabecera:

\begin{tabular}{|c|c|c|c|}
\hline $\begin{array}{c}\text { «...de la diagonal que por } \\
\text { tes restado y el ancho da..." } \\
(\mathrm{d} / \mathrm{u})^{2}\end{array}$ & "solución del ancho» & "solución de la diagonal & "Sus nombres» \\
\hline
\end{tabular}

n sólo sirve para numerar las quince líneas con números: $n=1$ a 15 .

En las 15 líneas $d^{2}-s^{2}$ es entero cuadrado perfecto: $d^{2}-s^{2}=u^{2}$.

La primera columna es $(\mathrm{d} / \mathrm{u})^{2}$. La operación indicada en la cabecera es, según Friberg, $(d / u)^{2}-1=\left(d^{2}-u^{2}\right) / u^{2}=(s / u)^{2}$.

Por tanto los valores que aparecen explícitamente son $(\mathrm{s}, \mathrm{d})$. Los valores $u$ aparecen de modo indirecto en la primera columna $(d / u)^{2}$. Dado que la tablilla está rota se supone que debería estar en esa parte.

Los triples pitagóricos $(\mathrm{d}, \mathrm{s}, \mathrm{u})$ a lo largo de las quince líneas suponen triángulos rectángulos cuyo ángulo $(d, u)$ va disminuyendo, desigualmente siempre un poco menos de $1^{\circ}$, desde algo menos de $45^{\circ}$ hasta algo más $31^{\circ}$. 


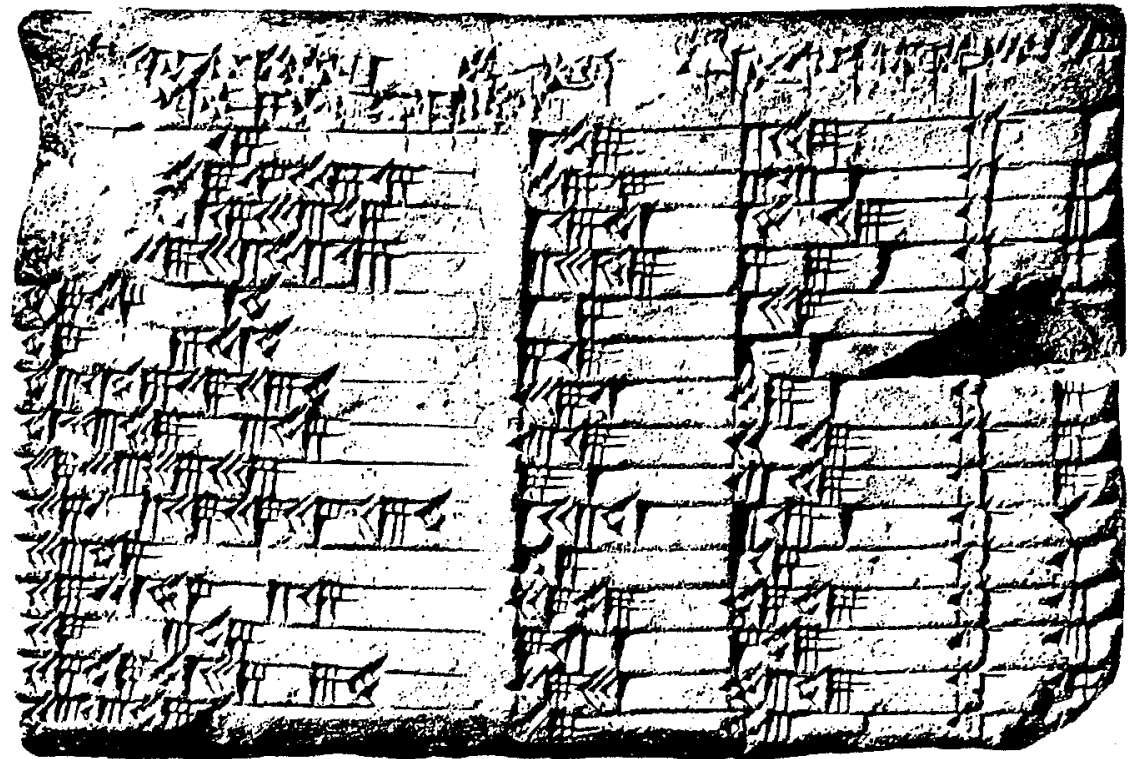

Plimpton 322

\begin{tabular}{|c|c|c|c|}
\hline Obverse & 11 & III & IV \\
\hline 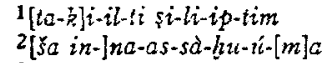 & $\left.\right|_{\text {sag } i-\ldots-i b} i_{b-s i g}$ sag & ib-sis $s i-l i-i p-i i m$ & mu-bi-im \\
\hline $3[1,59], 15$ & 1,59 & 2,49 & $k i-1$ \\
\hline $4[1,56,56], 58,14,50,6^{165}, 15$ & 56.7 & $3,12,1^{103}$ & ki-2 \\
\hline$[1,55,7], 41,15,33,45$ & $1,16,41$ & $1,50,49$ & $k i-3$ \\
\hline $6[1], 5[3,1] 0,29,32,52,16$ & $3,31,49$ & $5,9,1$ & $k i-4$ \\
\hline $7[1], \pm 8,54,1,40$ & 1,5 & 1,37 & $\left.\mathrm{ki}_{[}^{t}-5\right]$ \\
\hline $8[1]+47,6,41,10$ & 5,19 & 8,1 & {$[k i-6]$} \\
\hline $9[1], 43,11,36,28,26,40$ & 38,11 & 59,1 & $\mathrm{ki}-7$ \\
\hline $10[1], 41,33,59,3,45$ & 13,19 & 20,49 & $\mathrm{ki}-8$ \\
\hline $11[1], 38,33,36,36$ & $9,1^{106}$ & 12,49 & ki-9 \\
\hline $12[1], 35,10,2,28,27,24,26,40$ & $1,22,41$ & $2,16,1$ & ki-10 \\
\hline $13[1], 33,45$ & 45 & 1,15 & $k i-11$ \\
\hline${ }^{4}[1], 29,21,54,2,15$ & 27,59 & 48,49 & $\mathrm{ki}-12$ \\
\hline $15[1], 27,3,45$ & $7,12,1^{107}$ & 4,49 & ki-13 \\
\hline $16[1], 25,48,51,35,6,40$ & 29,31 & 53,49 & $\mathrm{ki}-14$ \\
\hline $17[1], 23,13,46,4[0]$ & 56 & $53^{100}$ & $k i[-15]$ \\
\hline
\end{tabular}




\section{CONCLUSIÓN}

La Matemática Paleobabilónica da solución a una gran cantidad de nuevos problemas matemáticos que ella misma enuncia, crea. Reconoce hechos matemáticamente ciertos en los cuales se interesa para darles forma. Para ello expresa las relaciones que ocurren entre los componentes del problema y comunica la solución mediante el nepešum (de epešum = hacer), el procedimiento, la solución del problema. Es evidente que el procedimiento paleobabilónico, el nepešum, resuelve ése problema numérico concreto ejemplificando la solución del problema para otros valores numéricos y tiene, de éste modo una generalidad.

La reiterada actividad en los textos paleobabilónicos en torno a la misma cuestión, dando en unos ejercicios unos datos y pidiendo el que falta y en otros ejercicios dando otros y pidiendo el que queda, revela el reconocimiento implícito en la Matemática Paleobabilónica de una relación fija entre las variables que componen el problema, la existencia de la matemática Paleobabilónica del concepto que actualmente denominamos fórmula. Sin embargo no existen los conceptos necesarios para describir las fórmulas de modo próximo al actual.

Atribuido a Tales (VI a.C.) 15 siglos después su teorema por referencia del texto, que tiene similitudes y diferencias con los Paleobabilónicos:

"Tras colocar un bastón en el límite de la sombra que proyecta la pirámide y formados dos triángulos por acción de los rayos del sol, (Tales) mostró que la relación que guarda ésta sombra con respecto a la otra es la que existe entre el bastón y la pirámide» ${ }^{26}$.

, similar a un procedimiento paleobabilónico pues igualmente aplica su teorema a la resolución de un problema práctico, el cálculo de la altura de una pirámide. La diferencia es que no lo ejemplifica con datos numéricos, $y$, a costa de no calcular efectivamente la altura de la pirámide, hace algo históricamente mejor, expresa una fórmula: «...la relación que guarda ésta sombra con respecto a la otra es la que existe entre el bastón y la pirámide».

La adquisición de los conceptos necesarios para describir fórmulas en vez de procedimientos es el gran avance que se percibe en éste texto sobre Tales. La propiedad matemática era conocida en la época Paleobabilónica.

26 «Los Filósofos Presocráticos 1». Pág. 73. ed. Gredos. 
"Pitágoras transformó la Filosofía concerniente a ella (esto es, a la geometría), en una forma de educación libre, examinando sus principios desde lo supremo e investigando los teoremas de un modo inmaterial e intelectual; ... ${ }^{27}$.

El enunciado del problema de Tales, en su intención de calcular la altura de una pirámide, no mejora enunciados paleobabilónicos; calcular la longitud de una cuerda a una profundidad dada sobre una circunferencia dada (BM 85194 \#20 página 93) es más «inmaterial e intelectual», más matemático. Los enunciados de los problemas clasificados (Nemet-Nejat CMTREM) en Algebra y Geometría lo son todos ellos; como ejemplo los problemas de algebra (págs. 83 a 87 ).

Pitágoras también da nombre a un teorema:

«El matemático Apolodoro dice que (Pitágoras) sacrificó cien bueyes cuando descubrió que, en el triángulo rectángulo, al cuadrarse la hipotenusa, (dicho cuadrado es) igual (a la suma de los cuadrados construidos sobre los lados) que circundan (al ángulo recto) $" 28$.

Como en los problemas Paleobabilónicos de Geometría y Algebra el objeto es inmaterial, matemático: «un triángulo rectángulo». A diferencia del enunciado de Tales, que quiere calcular la altura de una pirámide, el enunciado de Pitágoras no tiene más intención que expresar algo cierto matemáticamente: $d^{2}=u^{2}+s^{2}$ en los triángulos rectángulos.

A diferencia de los procedimientos paleobabilónicos, Pitágoras no pretende realizar un cálculo. Pero en la época paleobabilónica se ha utilizado el teorema de Pitágoras de modo evidente en triángulos rectángulos semejantes al de lados $(3,4,5)$. Sin demostar como tampoco lo demostró Pitágoras, que sepamos; tampoco Tales demostró su teorema.

Para Neugebauer, la Matemática Paleobabilónica permanece en una fase precientífica. Esta caracterización global, se complementa con una valoración concreta según la cual todo lo que en la matemática griega se atribuye a Tales, es herencia anterior y procede de la Matemática Paleobabilónica. También, buena parte de lo atribuido a Pitágoras ${ }^{29}$. Los procedimientos del Algebra Paleobabilónica, según Thureau-Dangin, en poco difieren de los actuales ${ }^{30}$.

\footnotetext{
27 «Los Filósofos Presocráticos 1». Pág. 184. ed. Gredos.

28 «Los Filósofos presocráticos 1», pág. 185. ed. Gredos.

29 O. Neugebauer «The Exact Sciences in Antiquity». Ed. Dover. Págs. 36 y 148.

30 F. Thureau-Dangin. SHSS. Pág. 136.
} 
La demostración nace en Grecia y, entendiendo la matemática de prueba como la verdaderamente científica, la Paleobabilónica, cuyos textos no contienen la demostración de las fórmulas que emplean, es precientífica. No en el sentido de que obtenga resultados erróneos, sino en el sentido que la Matemática Paleobabilónica tiene como fin primordial la aplicación de sus conocimientos y la creación de nuevas formulaciones en variadas situaciones. Encontramos en los textos Paleobabilónicos ejercicios que aplican correctamente la proporcionalidad de formas geométricas semejantes (Teorema de Tales), pero no encontraremos justificación alguna del teorema. También encontramos ejercicios correctamente resueltos, que aplican el Teorema de Pitágoras en situaciones claras a triángulos proporcionales a $(3,4,5)$. Pero no la demostración, o algún tipo de justificación, del teorema.

En la actualidad, se aprende, en la primera fase en la escuela, a multiplicar y dividir sin demostración previa. A aplicar la Ley de la Proporcionalidad y el Teorema de Pitágoras en variados ejercicios, sin haber realizado demostración de la ley y el teorema. Cálculos de áreas y volúmenes. La resolución de ecuaciones de primera grado, de segundo grado, de sistemas de dos ecuaciones con dos incógnitas, sin demostración de fórmulas y procedimientos empleados. De ése mismo modo, sin demostración, las Matemáticas aprendieron históricamente. La etapa Paleobabilónica es el primer momento de gran creatividad, de ella se suele destacar el Sistema Sexagesimal Posicional y el Algebra, olvidando la «riqueza y variedad» ${ }^{31}$ de su Geometría.

\section{BIBLIOGRAFIA Y ABREVIATURAS}

BAQIR, T. "Some more Mathematical texts from Tell Harmal». Sumer 7. 1951.

CAPLICE, R. «Introduction to Akkadian». 1988.

GANDZ, S. «The origin and development of cuadratic equations in Babylonian, Greek and early arabic Algebra". Osiris 3, 1937.

GOETZE, A. «A Mathematical Compendium from Tell Harmal». Sumer 7. 1951.

HAYES, J.L. «A Manual of Sumerian Grammar and Texts». 1990.

Neugebauer, O. «The Exact Sciences in Antiquity». 1957.

ThOMSEN, M.L. «The Sumerian Languaje». 1984.

MCT. «Mathematical Cuneiform Texts». O. Neugebauer y A. Sachs. 1945.

MKT. «Mathematische Keilschrift-Texte». O. Neugebauer. 1935.

SHSS. «Sketch for a History of the Sexagesimal System». F. Thureau-Dangin. Osiris 7. 1932.

SA. «Science Awakening". B.L. van der Waerden. 1954.

CMTREM. "Cuneiform Mathematical Texts as a Reflection of Everyday life in Mesopotamia». K.R. Nemet-Nejat. 1993.

Friberg. «Mathematik». Reallexikon der Assyriologie 7. Friberg. 1987-1990.

31 Friberg. Pág. 552. 